\title{
Estudio epidemiológico descriptivo de pacientes hospitalizados en el Servicio de ORL del Hospital Clínico de la Universidad de Chile entre los años 2007 y 2014
}

\author{
Descriptive epidemiological study of patients hospitalized in the \\ ENT Department of the Hospital Clínico de la Universidad de Chile \\ between the years 2007 and 2014 \\ M Constanza Domínguez $\mathbf{P}^{1}$, Alfredo Aguila $\mathbf{R}^{2}$, Natalia Cabrera $\mathbf{S}^{3}$, Rodolfo Nazar $\mathbf{S}^{3}$, \\ Eugenio Alzérreca $\mathrm{A}^{4}$.
}

\begin{abstract}
RESUMEN
Introducción: Las enfermedades de origen otorrinolaringológico (ORL) tienen una elevada prevalencia en atención primaria de salud, sin embargo, la información epidemiológica en pacientes hospitalizados es bastante escasa.

objetivo: Obtener características demográficas y prevalencia de enfermedades en Ios pacientes hospitalizados del Servicio de ORL del Hospital Clínico de la Universidad de Chile (HCUCh).

Material y método: Estudio de tipo descriptivo y retrospectivo, llevado a cabo en el Servicio de ORL del HCUCh, donde se revisaron los registros electrónicos, existentes de grupos relacionados a diagnóstico (GRD) de egresos, entre los años 2007 y 2014.

Resultados: Se incluyeron 7.353 egresos, con un promedio de edad de los pacientes de 28,24 años. La causa de hospitalización más frecuente fue la patología de faringe. Del total de los egresos $87,88 \%$ presentaron una intervención quirúrgica donde la amigdalectomía con adenoidectomía alcanzó el 15,7\% de las cirugías realizadas.

Conclusión: Este estudio nos entrega información epidemiológica sobre los pacientes hospitalizados en un servicio de ORL de un hospital de referencia a nivel nacional.

Palabras clave: GRD, egresos hospitalarios, patología otorrinolaringológica.
\end{abstract}

\section{ABSTRACT}

Introduction: Diseases of otolaryngology (ENT) origin have a high prevalence in primary health care, however, the epidemiological information on hospitalized patients is almost null.

\footnotetext{
Interno de Medicina. Universidad de Chile.

Médico Unidad de GRD, Hospital Clínico Universidad de Chile.

Servicio de Otorrinolaringología, Hospital Clínico Universidad de Chile.

4 Servicio de Otorrinolaringología, Hospital Guillermo Grant Benavente.
}

Recibido el 9 de noviembre de 2015. Aceptado el 28 de abril de 2016. 
Aim: To obtain demographic and disease prevalence information in hospitalized patients of the ENT Department at the Hospital Clínico de la Universidad de Chile (HCUCh).

Material and method: A descriptive and retrospective study, was carried out at the ENT department of the HCUCh, reviewing existing records of diagnostic related groups (GRD) for discharges between 2007 and 2014.

Results: We review a total of 7353 discharges, with an average age of patients of 28.24 years. The most frequent cause of hospitalization was pharynx pathology. An $87.88 \%$ of discharges had surgery performed, where tonsillectomy with adenoidectomy reached $15,7 \%$ of the total. Conclusion: This study provides us with epidemiological information on patients hospitalized in an ENT department in a nationwide referral hospital.

Key words: GRD, Hospital Discharges, ENT pathology.

\section{INTRODUCCIÓN}

Las enfermedades de oídos, nariz y laringe tienen una elevada prevalencia en nuestro medio, lo que se ve reflejado por los motivos de consultas en atención primaria de salud, donde se describe entre $40 \%$ y $50 \%$ de consultas diarias en el ámbito otorrinolaringológico ${ }^{1}$. Sin embargo, la evidencia que existe de estudios epidemiológicos en pacientes hospitalizados en servicios de otorrinolaringología en nuestro país es casi nula ${ }^{2}$. En el estudio epidemiológico de Ulloa y $\operatorname{cols}^{3}$, de un análisis de 8.275 egresos hospitalarios y 9.607 cirugías, se concluye que del espectro otorrinolaringológico, la patología faríngea es la más frecuente, con $40 \%$, seguida de la patología rinosinusal. El desglose por patología individual reveló que la principal causa de hospitalización y cirugía fue la enfermedad crónica de amígdalas y adenoides.

A nivel hospitalario, el servicio de otorrinolaringología tiene una actividad importante de consulta ambulatoria, mayor que la mayoría de otros servicios, debiendo generar atenciones en salud lo suficientemente costo-efectivas para cubrir las crecientes demandas de asistencia pública en recintos hospitalarios ${ }^{3,5}$. En relación a esto, la preocupación por lograr una distribución efectiva de recursos, surge como una necesidad imperiosa junto con el estudio de formas de organización sanitaria que puedan ofrecer mejoras en la eficiencia del sistema.

Los grupos relacionados por el diagnóstico (GRD), son una herramienta de gestión normalizadora, en la que mediante un registro electrónico, alimentado con datos de los egresos hospitalarios, podemos clasificar a los pacientes en grupos clíni- camente similares y con consumo semejante de recursos sanitarios, asignando pesos relativos al costo asociado de cada evento de hospitalización. Los GRD fueron creados con el objetivo de obtener una clasificación de episodios clínicos en función del consumo de recursos y manejo médico de los pacientes. El sistema GRD tiene una gran versatilidad y es capaz de satisfacer las necesidades individuales de un hospital particular, estableciendo bases para mejorar la gestión clínica, administrativa y financiera.

El Hospital Clínico de la Universidad de Chile (HCUCh) es un hospital universitario, que si bien no pertenece a la red de servicios públicos de salud, atiende gran cantidad de pacientes del área norte de Santiago de Chile y resto del país. Su servicio de otorrinolaringología está compuesto por 10 camas, cuenta con 2 pabellones quirúrgicos de funcionamiento diario y un policlínico de especialidad. Es centro de formación de postítulo en la especialidad, y continuamente recibe médicos de medicina general y otras especialidades en estadías de perfeccionamiento o capacitación.

\section{OBJETIVO}

En relación a lo expuesto, es de nuestro interés, analizar en este estudio descriptivo, el perfil epidemiológico de los pacientes hospitalizados en el Servicio de Otorrinolaringología del HCUCh, entre los años 2007 y 2014, con el objetivo de obtener datos actualizados sobre características demográficas y prevalencia de enfermedades, que nos permitan realizar una adecuada distribución de recursos. 


\section{MATERIAL Y MÉTODO}

Se realizó un estudio de tipo descriptivo y transversal, en el Servicio de Otorrinolaringología del HCUCh. Se revisaron los registros electrónicos de GRD de los egresos hospitalarios en el servicio desde el año 2007 hasta el año 2014, considerando para tal efecto un conjunto básico de datos, incluyendo aspectos demográficos (sexo y edad), y aspectos de tipo médico-hospitalario tales como causa principal de hospitalización, procedimiento principal realizado y la estadía hospitalaria en días. Estos datos de estadía promedio hospitalaria fueron cotejados con los datos existentes de estancia, según norma internacional para cada paciente, con el objetivo de calcular los días de estancia prolongada 0 outliers superior.

La aplicación de los GRD requiere conocer la casuística del hospital, o de un servicio o especialidad, y compararla con la de otro similar o con la de un conjunto de hospitales nacionales o extranjeros, con el objeto de obtener un marco de referencia llamado norma ${ }^{7}$. Conviene tener presente que se necesitan por lo menos 200.000 egresos hospitalarios con datos de buena calidad, para poder fijar una norma del hospital 7 . La estancia norma en este caso, fue definida como el promedio de días de estancia hospitalaria que tiene el conjunto del hospital, con el nivel de funcionamiento actual de éste. Se determinó como estancia media bruta la medida promedio de los días de estancia hospitalaria en pacientes del servicio en el periodo estudiado. Aquellos casos considerados atípicos o fuera de lo normal en cuanto a la estancia hospitalaria esperable para aquella patología, de acuerdo a la estancia norma, se denominan extremos o outliers. Se determina un límite superior de estancia para cada GRD, considerando los episodios que superen este límite como extremos.

El sistema de GRD establece un índice de severidad a cada grupo diagnóstico, basado en la exis- tencia o no de comorbilidades y/o complicaciones. Así se establecen 3 grupos:

Grupo 1: Sin comorbilidades y/o complicaciones.

Grupo 2: Con comorbilidades y/o complicaciones.

Grupo 3: Con comorbilidades y/o complicaciones mayores.

Las variables fueron descritas mediante frecuencias porcentuales, medianas y rangos obtenidos de datos.

\section{RESULTADOS}

Se revisaron los registros electrónicos existentes de GRD del Servicio de Otorrinolaringología del HCUCh entre los años 2007 y 2014, obteniendo un total de 7.353 egresos hospitalarios. Se consideró además la distribución de egresos finales por año y porcentaje de codificación GRD del total de fichas clínicas de pacientes hospitalizados en el servicio de ORL, con un promedio de codificación de 88,7, a la fecha de cierre de análisis de datos, en los 7 años analizados (Tabla 1).

En cuanto al perfil epidemiológico de los pacientes egresados del servicio, se obtuvo que 3.792 casos correspondieron a pacientes de sexo masculino $(51,5 \%)$, siendo el resto pacientes de sexo femenino (Tabla 2 y Figura 1). La edad promedio de los pacientes del servicio fue de 28,24 años.

Al analizar el tipo de ingreso de estos pacientes al hospital, se obtuvo que el $82,4 \%$ ingresaron en forma programada o electiva, mientras que $8,2 \%$ lo hizo de urgencia, ya sea a través del servicio de urgencia de nuestro hospital, o desde el policlínico de especialidad de ORL. En este último grupo, la causa más frecuente de hospitalización fue un absceso periamigdalino en el $36 \%$ de los casos.

Tabla 1. Distribución de egresos hospitalarios por año y porcentaje de codificación GRD

\begin{tabular}{|lrrrrrrrr|}
\hline & 2007 & 2008 & 2009 & 2010 & 2011 & 2012 & 2013 & 2014 \\
\hline Altas brutas & 955 & 915 & 788 & 907 & 874 & 928 & 1057 & 929 \\
\% codificación & 99,58 & 99,45 & 99,62 & 89,31 & 52,29 & 82,33 & 88,93 & 98,28 \\
\hline
\end{tabular}


Tabla 2. Distribución por sexo y edad de los pacientes ingresados al servicio de otorrinolaringología entre los años 2007 y 2014

\begin{tabular}{|c|c|c|c|c|}
\hline Edad & Hombres & Porcentaje & Mujeres & Porcentaje \\
\hline 0-14 años & 1.403 & $19 \%$ & 1.097 & $14,5 \%$ \\
\hline 15-44 años & 2.167 & $29,4 \%$ & 2.244 & $30,5 \%$ \\
\hline 650 más años & 222 & $3,0 \%$ & 220 & $2,9 \%$ \\
\hline Total & 3.792 & $51,5 \%$ & 3.561 & $48,4 \%$ \\
\hline
\end{tabular}

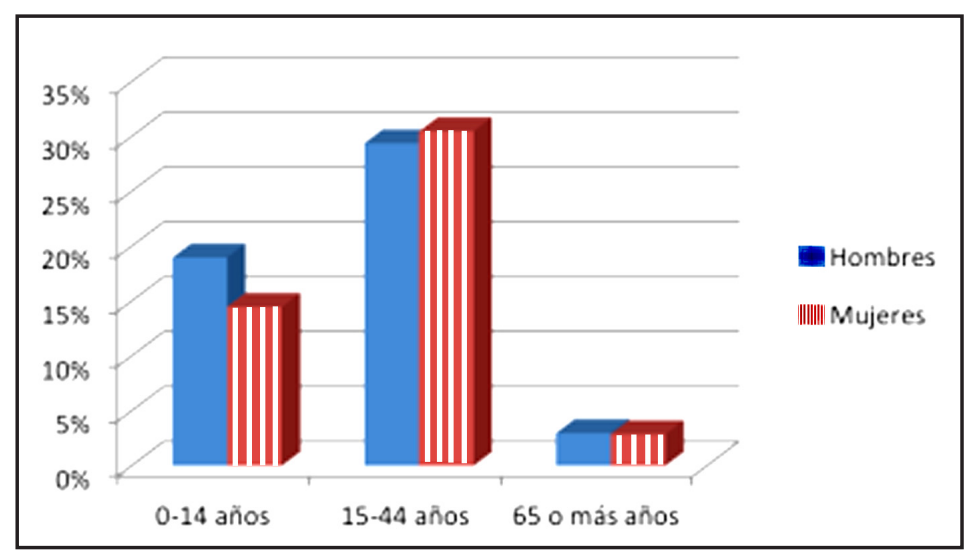

Figura 1. Distribución por sexo y edad de los pacientes ingresados al servicio de otorrinolaringología entre los años 2007 y 2014.

Con el objetivo de agrupar los diagnósticos de egreso, se les dividió arbitrariamente según sistema y región anatómica afectada, en patología de faringe y del anillo de Waldayer, patología nasosinusal, patología de oído y sistema vestibular, y patología de laringe y vía aérea, registrándose los 5 diagnósticos más frecuentes de cada grupo.

En cuanto a los diagnósticos principales consignados en los egresos codificados, se obtuvo que las principales causas de hospitalización fueron la patología de faringe, luego la patología de oído y vestibular, luego nasosinusal y finalmente patología de laringe (Tabla 3).

Al desglosar estos grupos, la patología adenoamigdalina constituyó el principal diagnóstico dentro de la patología faríngea; la otitis media no especificada en patología de oído y vestibular; la fractura de los huesos de la nariz en patología nasosinusal; y pólipo de cuerdas vocales en laringe (Tabla 4).
Con respecto a la actividad quirúrgica del servicio en estos años, se observó que el $87,88 \%$ de los egresos en GRD presentaron una intervención quirúrgica durante su hospitalización, utilizando horas de pabellón. Dentro de este grupo de pacientes las intervenciones más frecuentes correspondieron al grupo de patología faríngea, donde las amigdalectomías con 0 sin adenoidectomía alcanzaron el $21,5 \%$ de todas las intervenciones (Tabla 5).

La estancia norma calculada fue de 4,76 días, como promedio, y la estancia media bruta de 1,42 días. En nuestro hospital, el número total de egresos que presentaron una estadía prolongada en los años estudiados fueron 230, lo que corresponde al $3,1 \%$ del total de egresos hospitalarios (Tabla 6).

En nuestro servicio, la mayoría de los pacientes egresados se encuentra en nivel de severidad 1, lo que ubica al promedio de los egresos en un rango de baja complejidad (Tabla 7 ). 
Tabla 3. Diagnósticos CIE 10 más frecuentes servicio de otorrinolaringología entre los años 2007 y 2014

\begin{tabular}{|clr|}
\hline \multicolumn{2}{|c|}{ Diagnóstico GRD } & $\%$ \\
\hline 1. & Enfermedades crónicas de las amígdalas y adenoides (J35.X) & 29,3 \\
2. & Otros trastornos de la nariz y de los senos paranasales (J34.X) & 13,5 \\
3. & Otitis media supurativa y la no especificada (H66.X) & 7,0 \\
4. & Enfermedades de las cuerdas vocales y de la laringe, no clasificadas en otra parte (J38.x) & 4,5 \\
5. & Otoesclerosis (H80.x) & 4,2 \\
6. & Absceso periamigdalino (J36) & 4,1 \\
7. & Otitis media no supurativa (H65.X) & 3,3 \\
8. & Pólipo nasal (J33.X) & 3,0 \\
9. & Colesteatoma del oído medio (H71) & 2,7 \\
10. & Sinusitis crónica (J32.x) & 2,6 \\
\hline
\end{tabular}

Tabla 4. Patologías más frecuentes según área de otorrinolaringología entre años 2007 y 2014

\begin{tabular}{|c|c|c|}
\hline Área de otorrinolaringología & Patología & $\%$ \\
\hline Patología faríngea & $\begin{array}{ll}1 & \text { Hipertrofia de las amigdalas con hipertrofia de las adenoides } \\
2 & \text { Amigdalitis crónica } \\
3 & \text { Absceso periamigdalino } \\
4 & \text { Hipertrofia de las adenoides } \\
5 & \text { Hipertrofia de las amigdalas con hipertrofia de las adenoides }\end{array}$ & $\begin{array}{r}30,2 \\
13,2 \\
7,2 \\
3,9 \\
2,8\end{array}$ \\
\hline $\begin{array}{l}\text { Patología de oído y } \\
\text { vestibular }\end{array}$ & $\begin{array}{ll}1 & \text { Otitis media, no especificada } \\
2 & \text { Colesteatoma de oído medio } \\
3 & \text { Otras otitis medias crónicas no supurativas } \\
4 & \text { Otosclerosis, no especificada } \\
5 & \text { Otras otosclerosis }\end{array}$ & $\begin{array}{r}11,6 \\
4,7 \\
4,1 \\
4,1 \\
2,9\end{array}$ \\
\hline Patología nasosinusal & $\begin{array}{ll}1 & \text { Fractura de los huesos de la nariz-cerrada } \\
2 & \text { Otros pólipos de los senos paranasales } \\
3 & \text { Hipertrofia de los cornetes nasales } \\
4 & \text { Sinusitis crónica, no especificada } \\
5 & \text { Otros trastornos especificados de la nariz y de los senos paranasales }\end{array}$ & $\begin{array}{l}3,8 \\
2,9 \\
2,4 \\
2,2 \\
2,1\end{array}$ \\
\hline Patología laríngea & $\begin{array}{ll}1 & \text { Pólipo de las cuerdas vocales y de la laringe } \\
2 & \text { Tumor benigno de la laringe } \\
3 & \text { Otras enfermedades de las cuerdas vocales } \\
4 & \text { Otras enfermedades de la laringe } \\
5 & \text { Parálisis de las cuerdas vocales y de la laringe }\end{array}$ & $\begin{array}{l}3,8 \\
1,6 \\
1,0 \\
1,0 \\
1,0\end{array}$ \\
\hline
\end{tabular}

Tabla 5: Actividad quirúrgica del servicio de otorrinolaringología entre los años 2007 y 2014

\begin{tabular}{|clr|}
\hline \multicolumn{2}{|l|}{ Procedimiento } & $\%$ \\
\hline 1. & Amigdalectomía con adenoidectomía & 15,7 \\
2. & Otra septoplastía & 8,3 \\
3. & Amigdalectomía sin adenoidectomía & 5,8 \\
4. & Miringotomía con inserción de tubo & 5,2 \\
5. & Turbinectomía por diatermia o criocirugía & 5,1 \\
6. & Otros procedimientos diagnósticos sobre senos nasales (maxilares) & 4,6 \\
7. & Otra excisión o destrucción de lesión o tejido de la laringe & 3,5 \\
8. Miringoplastía & 3,1 \\
9. & Laringoscopía y otra traqueoscopía & 3,1 \\
10. & Etmoidectomía & 2,5 \\
\hline
\end{tabular}


Tabla 6. Estadías prolongadas o outliers entre los años 2007 y 2014

\begin{tabular}{|cccc|}
\hline Nivel de severidad & $N^{\circ}$ altas con outlier & Porcentaje de altas con outlier & Estancia media bruta (días) \\
\hline 1 & 214 & $93 \%$ & 5,84 \\
2 & 14 & $6,10 \%$ & 20,14 \\
3 & 2 & $0,90 \%$ & 26 \\
Total & 230 & $100 \%$ & 6,8 \\
\hline
\end{tabular}

Tabla 7. Distribución de egresos según nivel de severidad

\begin{tabular}{|ccc|}
\hline Nivel de severidad & Altas brutas & Porcentaje de altas \\
\hline No codificados en sistema GRD & 687 & $9,30 \%$ \\
1 & 6,247 & $85 \%$ \\
2 & 337 & $4,60 \%$ \\
3 & 82 & $1,10 \%$ \\
\hline
\end{tabular}

\section{DISCUSIÓN}

El concepto de GRD se origina a mediados de los años 60 en la Universidad de Yale, con el objetivo de crear un marco de referencia para evaluar la calidad de la asistencia a los pacientes y la utilización de los servicios prestados por los hospitales. El éxito desde entonces ha sido tal, que la mayoría de los países del mundo occidental utilizan los GRD como herramienta para evaluar el funcionamiento de sus hospitales. Los GRD se han ido perfeccionando con los años y se revisan sistemáticamente de acuerdo con los nuevos sistemas de clasificación y categorización de enfermedades, como el CIE-10 para los diagnósticos y CIE-9 MC para los procedimientos clínicos realizados.

En este artículo se describieron las características epidemiológicas de los pacientes hospitalizados en el Servicio de ORL del HCUCh entre los años 2007 y 2014, de acuerdo a los registros de egresos obtenidos de la oficina de GRD. Se logró obtener una codificación promedio de egresos en GRD del $88,7 \%$, lo que constituye un sesgo a la hora de analizar los datos obtenidos.

En concordancia con el resto de la literatura nacional, la patología faríngea fue la de mayor prevalencia en los pacientes hospitalizados en el servicio. Dentro de este subgrupo la hipertrofia amigdalina y la amigdalitis crónica ocupan el mayor porcentaje de diagnósticos en esta área, lo cual además concuerda con el resultado obtenido en número de procedimientos quirúrgicos realizados, donde la amigdalectomía con o sin adenoidectomía fue la cirugía más realizada ${ }^{3}$.

Dentro de la patología de oído, la otitis media crónica es la causa más común de hospitalización en este ámbito. Muchos de los pacientes en este grupo fueron hospitalizados para cirugía de timpanoplastía o cirugía radical de oído. Cabe destacar la alta prevalencia de otoesclerosis, lo cual podría ser explicado porque nuestro hospital recibe gran cantidad de pacientes derivados de distintos sectores del país para resolución quirúrgica, aunque no pertenece a la red pública de salud.

El índice de severidad de los pacientes del servicio fue bajo, lo que determina un bajo costo promedio de utilización de recursos. Sin embargo, es importante mencionar que la mayoría de los pacientes complejos del servicio son trasladados 0 manejados en intermedio médico o quirúrgico, lo que puede constituir un sesgo al evaluar el nivel de complejidad individual del servicio.

Otro de los estándares de calidad evaluados fueron las estadías prolongadas 0 outliers, que constituyeron el $3,1 \%$ del total de egresos. Este valor se encuentra por debajo del promedio del conjunto o total del hospital, y por debajo de otras especialidades médico-quirúrgicas.

El sistema de GRD tiene algunas limitaciones, en cuanto a que, sólo considera hospitalizaciones 
al alta, ya que está definido como un sistema de evaluación de pacientes en el egreso hospitalario, y por lo tanto, sólo se pueden modificar prácticas clínicas y administrativas en relación a pacientes hospitalizados, dejando de lado la atención ambulatoria, que representa gran parte de la actividad clínica del servicio.

La calidad de los análisis depende, en gran medida, de la calidad de los datos correctamente registrados por los clínicos tratantes, lo cual depende de la facilidad en el registro de diagnósticos, principal y secundarios, en la ficha clínica electrónica. Es relevante en estos casos, realizar un buen diagnóstico previo a implementar los GRD, a fin de determinar con precisión los puntos críticos y generar estrategias para solucionarlos. Es así también fundamental la capacitación adecuada del grupo o personal codificador ya que éste, es el otro pilar sobre el cual descansa la calidad final de los GRD.

\section{BIBLIOGRAFÍA}

1. Benito J, Morai D, Miyar V. Estudio descriptivo de la asistencia primaria en otorrinolaringología. Acta Otorrinolaringol Esp 1996; 47(1): 55-62.

2. Lechuga R, Frade C, Bahamonde P. Estudio epidemiológico descriptivo de una consulta de otología durante un año en un hospital de tercer nivel. An Otorrinolaringol Ibero Am 2002; 29 (2): 105-15.

3. Ulloa P, Urra A, Valenzuela A. Análisis de la atención intrahospitalaria del Servicio de Otorrinolaringología, Hospital Guillermo Grant Benavente de Concepción, 1993-1997. Rev Otorrinolaringol Cir Cabeza Cuello 1999; 59: 93-100.

\section{CONCLUSIÓN}

El sistema GRD es un potente complemento a las técnicas de administración en salud tradicionales, que permite focalizar el esfuerzo en la gestión clínica y financiera, para incrementar el nivel de control y seguimiento del impacto de decisiones relevantes de los equipos directivos de un hospital.

Los GRD permiten reconocer las diferentes prácticas asistenciales, lo que determina, en definitiva, las estrategias de mejoría de la calidad de atención al paciente. Esta herramienta posee una sólida capacidad de análisis de los aspectos administrativos, como de las variables médicas asociadas a un episodio clínico, lo que permite generar una gran cantidad de reportes analíticos que respaldarán una mejor gestión hospitalaria.

4. Greenfield S, Nelson C, Zubkoff M. Variations In resource utilization among medical specialties and systems of care. JAMA 1992; 267: 1624-30.

5. DeR C, Salin M, IÑIGUez M. Realidad de la derivación a otorrinolaringología desde la atención primaria. Rev Otorrinolaringol Cir Cabeza Cuello 2006; 66: 95-102.

6. Ruz S, Breinbauer H, Arancibia M. Análisis epidemiológico de la patología otorrinolaringológica ambulatoria en el Hospital San Juan de Dios. Rev Otorrinolaringol Cir Cabeza Cuello 2009; 69: 22732.

7. Álvareza M, Guerrero B, Ferrándiz R. Impacto de los grupos relacionados por el diagnóstico (GRD) en los medical devices. Rev Economia de la Salud 2005; 4: 115-8. 